\title{
Clubfoot pathoanatomy-biomechanics of deformity correction: a narrative review
}

\author{
Vincent S. Mosca ${ }^{1,2}$ \\ ${ }^{1}$ University of Washington School of Medicine, Seattle, WA, USA; ${ }^{2}$ Seattle Children's Hospital, Seattle, WA, USA \\ Correspondence to: Vincent S. Mosca, MD. Professor of Orthopedics, University of Washington School of Medicine; Pediatric Orthopedic Surgeon, Seattle \\ Children's Hospital, MB.10.623, PO Box 5371, 4800 Sand Point Way N.E., Seattle, WA 98105, USA. Email: vincent.mosca@seattlechildrens.org.
}

\begin{abstract}
Clubfoot is a common and complex, multi-segmental, 3-dimensional, congenital foot deformity. The segmental deformities have been recognized for millennia. They are cavus of the forefoot/midfoot, adductus of the midfoot, varus of the hindfoot, and equinus of the ankle, often referred to by the acronym CAVE. However, description of the deformities and their correction have varied over time with changes in consensus terminology for foot deformities and the understanding of foot biomechanics. In this narrative review, the most current terminology for foot deformities and understanding of foot biomechanics are applied to the clubfoot. The concept of the calcaneopedal unit (CPU) is applied to the in-depth discussion and understanding of clubfoot for perhaps the first time in the English language literature. Of the 4 basic clubfoot deformities, cavus and adductus are static structural deformities within the CPU, i.e., all bones of the foot except the talus. Varus is a restricted dynamic deformity between the CPU and the talus. Equinus is a restricted dynamic deformity in the ankle joint between the talus and the tibia. Understanding the CPU concept improves ones understanding of clubfoot deformities and the rationale for treatment techniques. Consensual agreement on the terms used to define interosseous relationships and motions in the foot improves communication. Finally, this narrative review highlights the histologic and biologic changes that occur in collagen and cartilage during clubfoot deformity correction to provide further understanding and rationale for treatment.
\end{abstract}

Keywords: Clubfoot; pathoanatomy; biomechanics; calcaneopedal unit (CPU); acetabulum pedis

Submitted Nov 16, 2020. Accepted for publication Apr 16, 2021.

doi: $10.21037 / \mathrm{atm}-20-7491$

View this article at: https://dx.doi.org/10.21037/atm-20-7491

\section{Introduction}

\section{Rationale/background}

Clubfoot is the most common congenital musculoskeletal deformity, affecting approximately 1 in 1,000 children around the world. It has been recognized for millennia. Hippocrates, the "Father of Medicine", wrote about management of clubfoot in $400 \mathrm{BC}$.

It is a complex, multi-segmental, 3-dimensional, congenital foot deformity. The segmental deformities have been recognized for centuries. They are cavus of the forefoot/midfoot, adductus of the midfoot, varus of the hindfoot, and equinus of the ankle, often referred to by the acronym CAVE. However, description of the deformities and their correction have varied over time with changes in consensus terminology for foot deformities and the understanding of foot biomechanics.

This issue of Annals of Translational Medicine consists of personal experiences in the management of clubfoot deformities by a group of international expert practitioners and researchers in the field.

\section{Objectives}

This narrative review was written to serve as a foundation for understanding the clubfoot deformity and its management based on the most current consensus terminology for foot deformities and understanding of 
foot biomechanics. Such a foundation is required to put the treatment outcomes in perspective. I present the following article in accordance with the Narrative Review reporting checklist (available at https://dx.doi.org/10.21037/atm-20-7491).

\section{Methods}

English language texts and articles pertaining to foot biomechanics and terminology that were published in the last 30 years were reviewed for the preparation of this manuscript.

\section{Narrative discussion}

Like developmental hip dysplasia and idiopathic scoliosis, clubfoot is a developmental deformation and not an embryonic malformation. Malformation means "made wrong". Simplistic terms for the five categories of malformations are too large, too small, too many, too few, and failed to separate (1). Deformation, or deformity, means that the bones and joints are normally formed but they are mal-aligned. When skeletally immature, especially very immature, bones and joints are mal-aligned for a protracted period, they change shape according to the Heuter-Volkmann property of growing cartilage. The mild changes in shape in clubfeet are, therefore, secondary to the deformities and do not reclassify them as malformations. They weren't made wrong. Deformations can develop any time after joints are created in the $7^{\text {th }}$ to $9^{\text {th }}$ weeks of gestation. In the case of clubfoot, the malalignment occurs in the second trimester of pregnancy. Clubfoot is rarely detected with ultrasonography before the 16th week of gestation (2).

Malformations can never be made normal. Deformations also cannot be made normal, but they can approach normal with effective and timely treatment. Normal feet can be expected to have long term comfort, function, and appearance. Dr. Ponseti showed that his method can convert clubfeet into feet that are comfortable, functional, and "normal" in appearance for decades. In order to replicate his excellent documented results, one must fully understand and practice his method without omissions or modifications, because strict adherence to all aspects of the method is mandatory. This starts with a full and complete understanding of the biomechanics of the clubfoot deformity and deformity correction.

Dr. Ponseti identified the clubfoot deformities as pronation of the forefoot (producing cavus), adduction of the midfoot (adductus), inversion of the subtalar joint/ hindfoot (varus), and plantar flexion of the talus in the ankle (equinus), thereby creating the acronym CAVE. And he listed the orderly correction of those deformities as first supinating the pronated forefoot and then abducting the adducted midfoot. But then he used terms that, based on current generally accepted terminology and understanding of biomechanics, are confusing when he stressed that the subtalar joint inversion should be corrected by "Lateral displacement (abduction) of the calcaneus to its normal relationship with the talus" (2). In fact, there is no adduction or abduction in the subtalar joint. There are only inversion and eversion. And Dr. Ponseti strongly and specifically cautioned against everting the subtalar joint that he acknowledged was inverted.

Current generally accepted terminology for foot deformities and the biomechanics of foot motions are based on the concept of the CPU (3). I believe it is important to translate Dr. Ponseti's terminology into the new, modern terminology to make it relevant and consistent with that used for all other foot deformities. This translation does not in any way change or diminish Dr. Ponseti's correct concepts. It merely changes some of the words used.

The CPU refers to all bones of the foot, except the talus (and excluding the toes) (Figure 1). Whereas static angular and rotational deformities may exist between the bones within the CPU, there is very little movement between those bones. The major motions within the foot occur between the CPU and the talus. The smaller and most important segmental unit within the CPU is the acetabulum pedis (AP), a term coined by Scarpa in 1818 (4). It consists of the bones and ligaments of the CPU immediately adjacent to the talus, i.e., the proximal articular surface of the navicular, the spring ligament, and the anterior/middle facets of the calcaneus (Figure 2).

The "joint" between the CPU/AP and the talus is referred to as the subtalar joint complex (STJC). The subtalar joint, strictly speaking, is the talo-calcaneal joint (sub $=$ under the talus). But the calcaneus cannot move relative to the talus without the navicular, cuboid, and the rest of the CPU moving with it, because the CPU is an extension of the calcaneus. The STJC inverts and everts around the talus following the oblique axis of the subtalar joint. Inversion is a combination of internal rotation, plantar flexion, and supination of the CPU/AP around the talus. Eversion is a combination of external rotation, dorsiflexion, and pronation between those two parts. The CPU/AP can only move around the fixed oblique axis of the STJC which is the same in normal feet and in those with hindfoot deformities. The STJC is in an exaggerated 

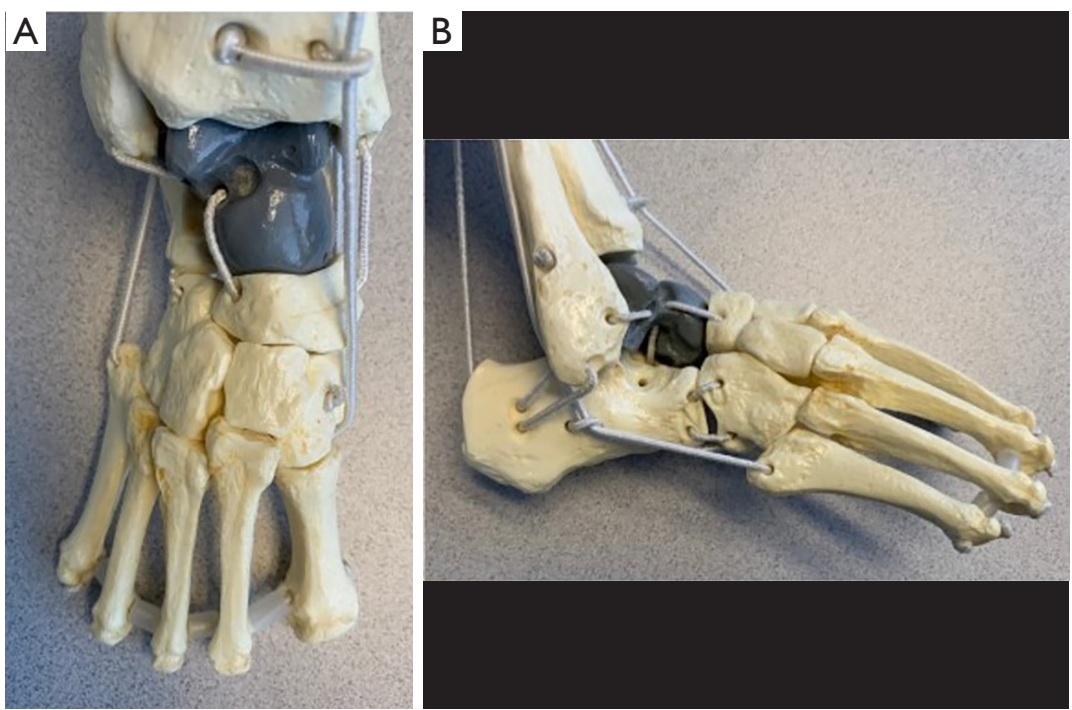

Figure 1 The calcaneopedal unit (CPU) is the term for all bones of the foot (white in color in this model made by John Mitchell and available through MD Orthopaedics, Inc.) except the talus (gray in color). The CPU and its inner-most component, the acetabulum pedis, inverts and everts around the talus through the subtalar joint complex. (A) Frontal view of foot model; (B) lateral view of foot model.

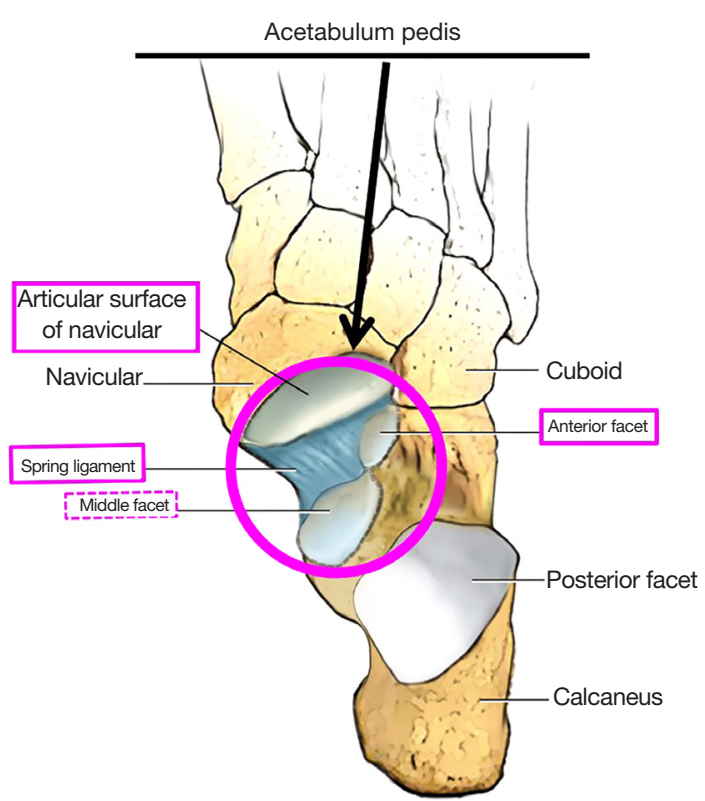

Figure 2 The acetabulum pedis (AP) [from: Mosca VS (1), figures 2-5, page 8].

and fixed inverted position in clubfoot and cavovarus foot deformities. It is in an exaggerated everted position in flatfoot and skewfoot deformities. Individual components of inversion and eversion cannot be isolated.

Ponseti was correct when he stated that one should not attempt to correct the hindfoot varus deformity until the forefoot pronation and midfoot adduction deformities are corrected. Kite's method of cast-correction of clubfoot deformities involved pronating the already pronated forefoot (5) and it was unsuccessful. The reason might relate to the postmortem fetal clubfoot dissection findings of Epeldegui (6,7). He found that the calcaneo-navicular spring ligament was contracted in clubfeet thereby resulting in a smaller than normal $\mathrm{AP}$, although this is my interpretation of his findings and not his stated conclusion. Perhaps that small AP cannot rotate around the larger head of the talus. And perhaps supinating the forefoot and abducting the midfoot with serial casting, as recommended by Ponseti, allow the spring ligament to subsequentially stretch because it is at the central core of those more superficial deformities. Stretching the spring ligament would enlarge the AP enabling it to rotate around the head of the talus when counter-pressure is applied to the dorsolateral aspect of the head of the talus.

Forefoot pronation and hindfoot varus are rotationally opposite direction deformities, as if the foot is wrung out like a towel (1). Forefoot pronation is a static rotational deformity within the CPU, i.e., the forefoot is pronated relative to the sagittal plane of the calcaneus (Figure $3 \mathrm{~A}$ ). Hindfoot varus is a static angular deformity of the calcaneus in relation to the tibia that is due to inversion of the subtalar joint. And inversion is a dynamic rotational and 

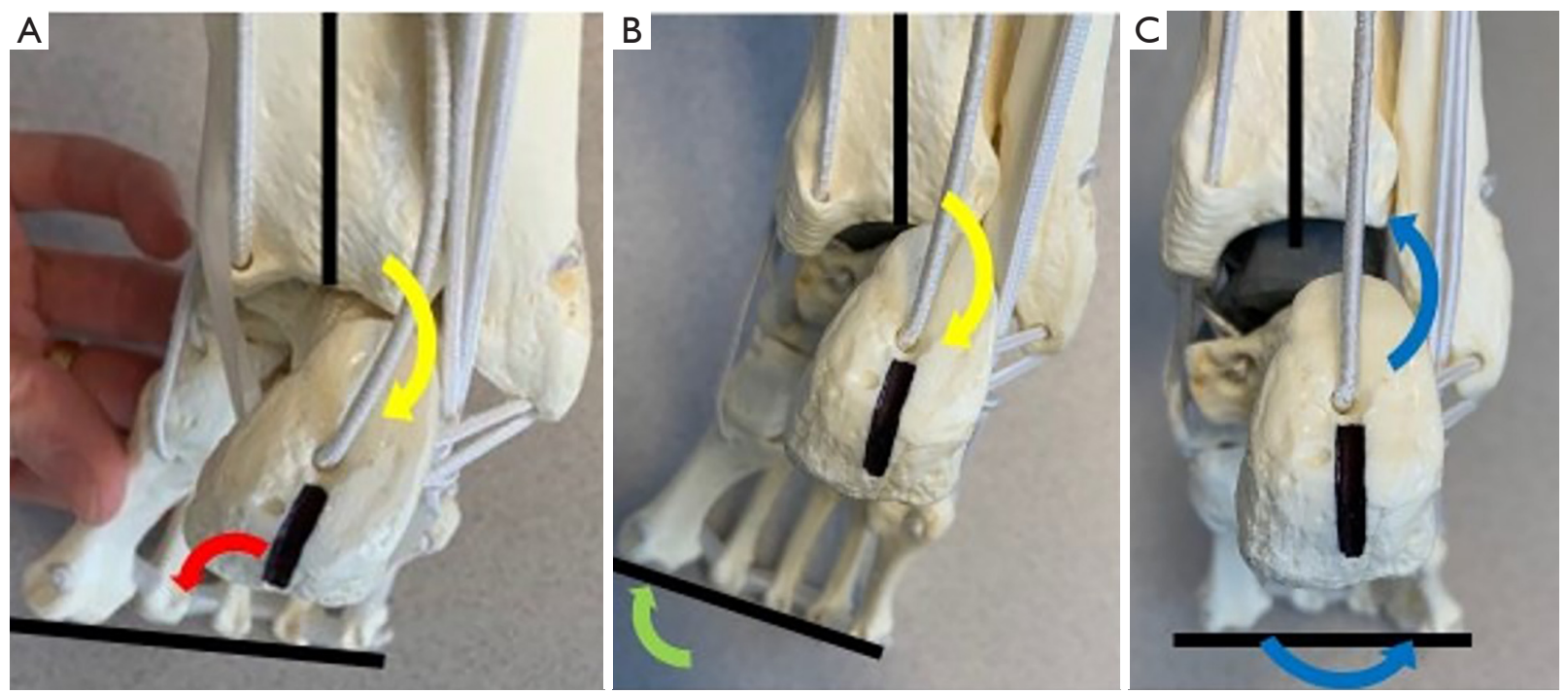

Figure 3 Clubfoot model. (A) The forefoot is pronated in relation to the hindfoot. Pronation is an interosseous deformity between the bones in the calcaneopedal unit (CPU). The transverse plane of the metatarsal heads (black line under the MT heads) is pronated (red curved arrow) in relationship to the sagittal plane of the calcaneus (short black line). Hindfoot varus is the static alignment between the calcaneus and the talus/tibia due to inversion of the subtalar joint (yellow curved arrow). (B) Supination molding of the forefoot (green curved arrow) corrects the pronation deformity within the CPU resulting in the normal perpendicular relationship between the transverse plane of the metatarsal heads (black line under the MT heads) and the sagittal plane of the calcaneus (short black line). This correction is usually achieved after the 1 st or 2 nd cast. Then, the only residual deformity in the clubfoot (not including the equinus in the ankle joint) is inversion/varus deformity of the subtalar joint (yellow curved arrow). (C) Eversion molding of all the bones of the CPU as a single unit (blue curved arrows) under and around the talus in the subtalar joint complex completes correction of all deformities within the clubfoot-leaving only ankle equinus deformity which, in most cases, requires a tendo-Achilles tenotomy. Note that the relationship between the transverse plane of the metatarsal heads (black line under the MT heads) and the sagittal plane of the calcaneus (short black line) within the CPU does not change, i.e., the bones and joints within the CPU remain anatomically aligned in this last stage of deformity correction. Plantar flexion/ internal rotation (inversion) deformity of the CPU corrects to dorsiflexion/external rotation (eversion).

angular deformity between the CPU and the talus in the STJC. Correcting forefoot pronation (a static rotational deformity within the CPU) and midfoot adduction (a static angular deformity within the CPU) in the first one or two casts creates normal interosseous alignment between all the bones and joints within the CPU. This is manifest by a perpendicular relationship between the transverse plane of the metatarsal heads, as a proxy for the anterior CPU, and the sagittal plane of the calcaneus (Figure 3B). Then the only residual deformity, other than equinus, is inward rotation/plantar flexion (inversion) of the CPU under the talus. With stretching of the spring ligament and enlargement of the AP, the subtalar joint can be safely everted, not abducted, around the talus. The "apparent" forefoot supination deformity that is intentionally created by casting reverts to neutral as the subtalar joint is everted. When the subtalar joint is fully everted, the forefoot is in neutral rotational alignment relative to the tibia and the hindfoot, neither pronated nor supinated (Figure 3C). The point is that eversion is not a dirty word. It's the current universally accepted term for correction of subtalar joint inversion deformity regardless of etiology, whether congenital or acquired.

The central structural axis of rotation of the STJC is the talocalcaneal interosseous ligament that is located between the middle and posterior facets (1). The clubfoot manipulations, as described by Ponseti, align the anterior aspect of the STJC by rotating the navicular dorso-laterally around the head of the talus (Figure $4 A$ ). At the same time, the calcaneo-fibular ligament stretches to enable the posterior aspect of the STJC to passively rotate plantarmedially away from the fibula based on the central position of the interosseous ligament and the oblique axis of the subtalar joint (Figure 4A). And the hand positions that Dr. 


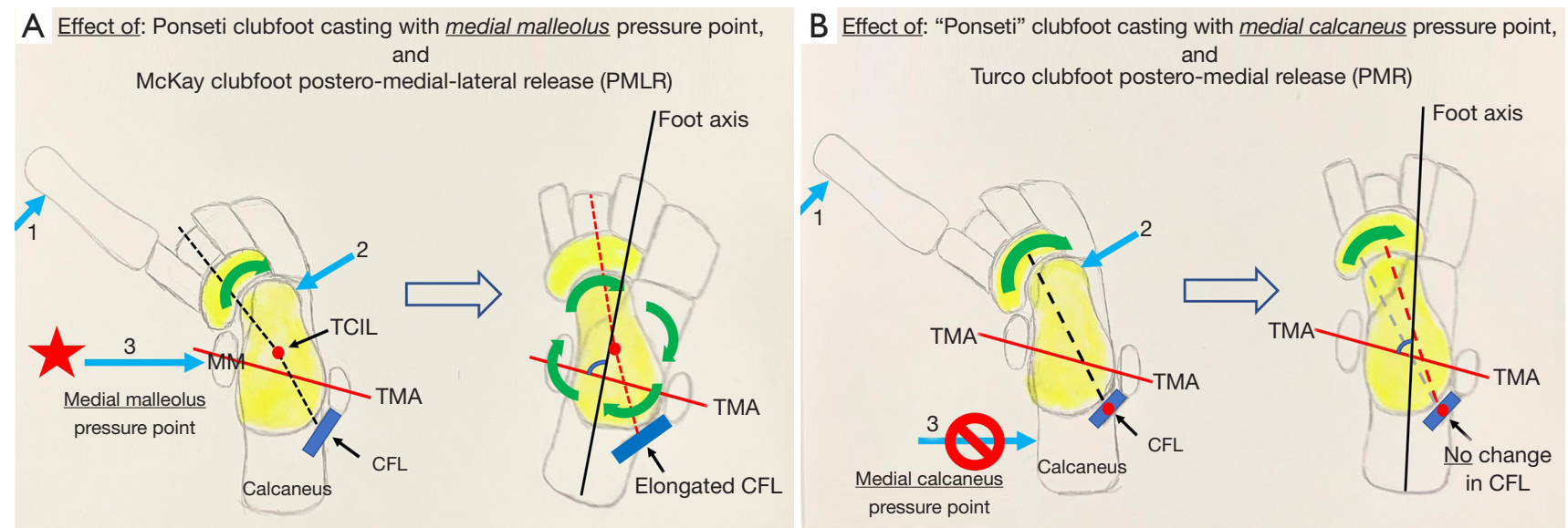

Figure 4 Clubfoot deformity correction. (A) The central point of rotation along the axis of the subtalar joint around which the subtalar joint complex (STJC) rotates is the talocalcaneal interosseous ligament (TCIL—red dot). It is located between the middle and posterior facets of the subtalar joint. According to Ponseti, the 3 pressure points (blue arrows) for manipulations and casting are the plantar-medial side of the 1st metatarsal head [1], the dorso-lateral head of the talus [2], and the medial malleolus [3]. Alignment of the anterior aspect of the STJC is produced by everting (green curved arrow) the calcaneopedal unit (CPU) around the head of the talus after the pronation and adduction deformities within the CPU have been corrected. Due to the relative size of the segments, the manipulations are, by necessity, anterior to the interosseous ligament rotation point. The medial malleolus pressure point allows the calcaneo-fibular ligament (CFL) to stretch and the posterior aspect of the STJC to rotate plantar-medially away from the fibula. The black dotted lines convert to the red dashed lines following deformity correction by means of an external rotation spin (green curved arrows) around that central axis of the subtalar joint. The transmalleolar axis (TMA) is drawn as a reference line to show correction of the internal rotation deformity in the STJC to a normal transmalleolar angle (blue curved angle line between the foot axis and the TMA). The McKay postero-medial-lateral surgical clubfoot release (PMLR), that includes release of the CFL, accomplishes the same goal, but at the cost of stiffness and eventual pain. (B) Pressure on the medial aspect of the calcaneus (blue arrow covered with "no symbol"), rather than the medial malleolus, prevents the CFL from stretching and the calcaneus from rotating away from the fibula. With this pressure point on the medial calcaneus, the center of rotation of the STJC shifts to the CFL (red dot on CFL), thereby changing spin of the joint around the interosseus ligament to hinging of the joint on the CFL. The talocalcaneal interosseous ligament then either stretches inappropriately or the cartilage anlage of the talus and calcaneus on the lateral side of the talocalcaneal joint compress according to the Heuter-Volkmann property of growing cartilage. The dashed red line represents an iatrogenically created axis in the near-sagittal plane around which the calcaneus can rotate in the coronal plane, effectively creating true (though undesired) "abduction" of the subtalar joint. Note the persistent inward rotation of the foot in relation to the TMAthe transmalleolar angle between the Foot axis and the TMA. The Turco postero-medial surgical clubfoot release (PMR), by ignoring and failing to release the postero-lateral corner, results in the same false correction as the improper casting technique. Like the McKay procedure, the Turco procedure creates stiffness and eventual pain, but without even truly correcting the deformity.

Ponseti so clearly and accurately described allow that to occur exactly as he proved decades ago (2). The subtalar joint can only move around its axis of rotation and in no other way unless its motions are inappropriately restricted externally. That happens if counter pressure is applied to the medial side of the calcaneus rather than the medial malleolus, a point stressed by Dr. Ponseti. Counter pressure on the medial side of the calcaneus prevents stretching of the contracted calcaneo-fibular ligament and rotation of the calcaneus away from the fibula. The center of rotation then shifts from the talocalcaneal interosseus ligament to the calcaneo-fibular ligament thereby creating false correction of the STJC deformity (Figure 4B).

The talonavicular joint will usually align whether the posterior point of counter pressure is the medial malleolus or the medial side of the calcaneus. But the CPU will only externally rotate on the interosseous ligament to the normal anatomic position under the talus if the posterior point of counter pressure is the medial malleolus. This is analogous to the McKay postero-medial-lateral clubfoot surgical release (PMLR) in which he stressed the importance of releasing the contracted calcaneo-fibular ligament (8). A 
positive, or outward, thigh-foot angle is achieved with the Ponseti non-operative and the McKay operative techniques (Figure $4 A$ ). Think of this as untwisting a cap on a bottle where the center of rotation is the center of the neck of the bottle. Alternatively, counter pressure on the medial side of the calcaneus will result in persistent inward rotation of the CPU despite alignment of the talonavicular joint because the center of rotation becomes the calcaneofibular ligament. This is analogous to the Turco posteromedial surgical clubfoot release (PMR) in which he failed to address the postero-lateral corner (9). A negative, or inward, thigh-foot angle results from the Kite non-operative and the Turco operative techniques (Figure 4B). Think of this as changing rotation on a central axis to the opening of a book in which the calcaneo-fibular ligament is the spine of the book. The Kite non-operative and Turco operative techniques effectively create a coronal plane hinge joint that rotates around a sagittal plane axis thereby enabling non-physiologic "abduction" of the hindfoot. I only mention these surgical procedures to help explain the anatomy and pathoanatomy and not to promote the surgical management of clubfeet.

It is important to understand the biologic and histologic changes that occur in clubfoot collagen and cartilage during deformity correction with the Ponseti method.

Creep is the term for the viscoelastic property of collagen that enables it to elongate when it is subjected to a constant load (10). The 30-60 seconds of manipulation and stretching immediately before each cast application take advantage of that property. The crimping of the collagen fibers is diminished. The collagen is longer and the deformities are improved at the end of the manipulation. There is a finite, yet unpredictable, amount of stretch/ elongation of collagen that can occur at any point in time. The goal is to stretch the collagen and not tear or rupture it. That's the reason Dr. Ponseti stressed that the child should not cry during manipulation of the foot. Of course, babies cry for many reasons, but the goal is to stretch the collagen gently so as not to cause pain-inducing tissue damage with its associated bleeding and scar formation.

Stress relaxation is the term for the viscoelastic property of collagen that enables it to gradually elongate after a static maximum load has been applied (10). The collagen relaxes/ elongates over time, thereby decreasing the effective load that was applied. A clubfoot cast is applied after creep has maximized collagen elongation at that moment in time. At the time of cast removal several days later, the collagen is longer and the deformities are improved compared with their appearance at the time of cast application. That's stress relaxation. It is not known exactly how long it takes for the collagen to reach its maximum passive elongation. Most pediatric orthopedic surgeons, like myself, change the casts weekly, based on our clinic schedules rather than the clubfoot biology. The efficacy and safety of shorter intervals between casts have been reported by some authors. At the time of the cast change, the collagen in the tendons, ligaments, and muscles is longer than it was. Unlike a rubber band that returns to its original length even after being held in an elongated position for a protracted period of time, the collagen with its elastic fibers is longer-a longer "rubber band" that is serially subjected to creep and stress relaxation from a new starting length each time the cast is changed. Of course, without the application of a cast after manipulation, the collagen resumes its former length. And after full deformity correction has been achieved, full time and then part time brace wear is necessary to maintain maximum stretch on the collagen and prevent recurrent deformity. This is particularly important during the first 3-4 years of life when excessive collagen synthesis in the ligaments, tendons, and muscles persists and is a known cause of relapses.

There is one other biologic change that takes place during Ponseti clubfoot management and it relates to the shapes of the bones and orientation of the joints. Pirani (11) showed this beautifully in his serial magnetic resonance imaging (MRI) monitoring of feet undergoing Ponseti treatment. The mild misshapenness of the navicular and talus correct to normal as the navicular is rotated to its anatomic alignment on the head of the talus. The varus/ adducted mal-orientation of the calcaneo-cuboid joint corrects to anatomically normal orientation with reshaping of the adjacent bone ends. This is due to the HeuterVolkmann property of growing cartilage. Although unproven, these changes are likely more permanent than those in the collagen due to the excessive collagen synthesis in the ligaments, tendons, and muscles that occurs in the first 3-4 years of life.

The tendo-Achilles, unlike the tarsal ligaments and the other plantar-medial tendons that are stretchable, is made of non-stretchable, thick, tight collagen bundles with few cells. It rarely stretches completely even with skillful Ponseti casting, most often requiring a tenotomy to complete correction of the equinus deformity (2). Care must be exercised to avoid over-stretching the plantar-medial soft tissues and creating a rocker-bottom foot deformity in an attempt to fully stretch the unyielding tendo-Achilles. Percutaneous tendo-Achilles tenotomy is needed in almost all clubfoot deformities following the 5-6 casts that are required to correct the other deformities. 
The last cast is left in place for 3 weeks while the severed heelcord tendon regenerates filling the gap between the tendon ends at the proper length and with minimal.

\section{Summary}

Clubfoot is the most common congenital musculoskeletal deformity around the world. Historical surgical treatment resulted in stiff, painful, poorly functioning and, often, multioperated feet. With the Ponseti non-operative method of clubfoot management, children can expect to have feet that are flexible, comfortable, strong, and functional for decades. The success of the Ponseti method is based on a provider's thorough understanding of the biomechanics of the foot and the pathoanatomy of the clubfoot deformity. Agreed upon terminology of foot deformities aids with communication between health care providers. The intent of this narrative review was to update the current terminology for foot deformities and the understanding of foot biomechanics to aid with that educational goal.

\section{Acknowledgments}

Funding: None.

\section{Footnote}

Provenance and Peer Review: This article was commissioned by the Guest Editors (Federico Canavese and Alain Dimeglio) for the series "Clubfoot" published in Annals of Translational Medicine. The article has undergone external peer review.

Reporting Checklist: The author has completed the Narrative Review reporting checklist. Available at https://dx.doi. org/10.21037/atm-20-7491

Conflicts of Interest: The author has completed the ICMJE uniform disclosure form (available at https://dx.doi. org/10.21037/atm-20-7491). The series "Clubfoot" was commissioned by the editorial office without any funding or sponsorship. The author has no other conflicts of interest to declare.

Ethical Statement: The author is accountable for all aspects of the work in ensuring that questions related to the accuracy or integrity of any part of the work are appropriately investigated and resolved.
Open Access Statement: This is an Open Access article distributed in accordance with the Creative Commons Attribution-NonCommercial-NoDerivs 4.0 International License (CC BY-NC-ND 4.0), which permits the noncommercial replication and distribution of the article with the strict proviso that no changes or edits are made and the original work is properly cited (including links to both the formal publication through the relevant DOI and the license). See: https://creativecommons.org/licenses/by-nc-nd/4.0/.

\section{References}

1. Mosca VS. Principles and Management of Pediatric Foot and Ankle Deformities and Malformations. Philadelphia, PA: Wolters Kluwer/Lippincott Williams \& Wilkins, 2014.

2. Ponseti IV. Congenital Clubfoot: Fundamentals of Treatment. Oxford: Oxford University Press, 1996.

3. Ghanem I, Massaad A, Assi A, et al. Understanding the foot's functional anatomy in physiological and pathological conditions: the calcaneopedal unit concept. J Child Orthop 2019;13:134-46.

4. Scarpa A, Peltier LF. A Memoir on the Congenital Club Feet of Children, and of the Mode of Correcting That Deformity. Clin Orthop Relat Res 1994;308:4-7.

5. Kite J. The Clubfoot. New York, NY: Grune \& Stratton, 1964.

6. Epeldegui T, Delgado E. Acetabulum pedis. Part I: Talocalcaneonavicular joint socket in normal foot. J Pediatr Orthop B 1995;4:1-10.

7. Epeldegui T, Delgado E. Acetabulum pedis. Part II: Talocalcaneonavicular joint socket in clubfoot. J Pediatr Orthop B 1995;4:11-6.

8. McKay DW. New concept of and approach to clubfoot treatment: section I-principles and morbid anatomy. J Pediatr Orthop 1982;2:347-56.

9. Turco VJ. Surgical correction of the resistant club foot. One-stage posteromedial release with internal fixation: a preliminary report. J Bone Joint Surg Am 1971;53:477-97.

10. Nordin M, Frankel V. Basic Biomechanics of the Musculoskeletal System. 2nd edition. Philadelphia: Lea \& Febiger, 1989.

11. Pirani S, Zeznik L, Hodges D. Magnetic resonance imaging study of the congenital clubfoot treated with the Ponseti method. J Pediatr Orthop 2001;21:719-26.

Cite this article as: Mosca VS. Clubfoot pathoanatomybiomechanics of deformity correction: a narrative review. Ann Transl Med 2021;9(13):1096. doi: 10.21037/atm-20-7491 